\title{
Making an accurate diagnosis of chronic hypersensitivity pneumonitis
}

\author{
Kerri A Johannson $\mathrm{MD}^{1,2,3}$, Christopher J Ryerson $\mathrm{MD}^{4}$
}

\begin{abstract}
KA Johannson, CJ Ryerson. Making an accurate diagnosis of chronic
\end{abstract} hypersensitivity pneumonitis. Can Respir J 2014;21(6):370-372.

Chronic hypersensitivity pneumonitis (HP) arises from repeated exposure to causative antigens. Although HP can be challenging to diagnose, it is important to differentiate from idiopathic pulmonary fibrosis and idiopathic nonspecific interstitial pneumonia. HP has a unique management approach and portends a unique prognosis. The present article summarizes the recent published literature on chronic HP and highlights the features that may be helpful in distinguishing it from other chronic interstitial lung diseases.

Key Words: Hypersensitivity pneumonitis; Interstitial lung disease; Review hronic hypersensitivity pneumonitis (HP) is an interstitial lung disease (ILD) that arises in a sensitized individual after sustained or repeated exposure to an inhaled antigenic stimulus. It can progress to end-stage fibrosis and shares many clinical and radiological features with idiopathic pulmonary fibrosis (IPF) and idiopathic nonspecific interstitial pneumonia (NSIP). The purpose of the current focused review is to present recent advances in the understanding of chronic HP while highlighting the features differentiating it from IPF and idiopathic NSIP.

\section{EPIDEMIOLOGY}

HP comprises between $4 \%$ and $15 \%$ of all ILDs, with higher proportions reported at tertiary referral centres (1). The true prevalence of $\mathrm{HP}$ is unknown due to its under-recognition, as well as geographical and temporal variation related to the spatial distribution of inciting environmental exposures. It is estimated that $<15 \%$ of individuals exposed to high levels of causative agents develop HP and $<3 \%$ of all farmers develop farmer's lung (1).

\section{CLINICAL MANIFESTATIONS AND DIAGNOSIS}

HP patients are typically younger than IPF patients, more likely to be female and less likely to have a history of cigarette smoking (2) (Table 1). Patients typically present with cough and exertional dyspnea that may improve during periods away from the inciting antigen. Hypoxemia, clubbing, auscultatory crackles and findings of cor pulmonale may be present. Pulmonary function tests commonly demonstrate restriction with a reduced diffusion capacity, although concomitant obstruction can occur. Importantly, there are no highly specific features, requiring consideration of HP whenever an ILD is encountered.

Identifying an antigen is a key component of the clinical evaluation. The most common causes of HP include exposure to bird feathers and droppings, mold, fungi and farming; however, $>300$ sources of antigen have been reported $(1,3)$. Several hours of daily exposure is typically required to cause HP; however, coincidental exposures may occur in other ILDs (4). Conversely, a culprit antigen is not identified in up to $50 \%$ of HP patients despite detailed assessment (2,5), possibly

\section{Poser un diagnostic précis de pneumopathie d'hypersensibilité chronique}

La pneumopathie d'hypersensibilité (PH) chronique découle d'une exposition répétée à des antigènes causaux. Même si la $\mathrm{PH}$ peut être difficile à diagnostiquer, il est important de la distinguer de la fibrose pulmonaire idiopathique et de la pneumopathie interstitielle diffuse idiopathique. La PH s'associe à une prise en charge unique et présage un pronostic unique. Le présent article résume les publications récentes sur la $\mathrm{PH}$ chronique et fait ressortir les caractéristiques susceptibles d'être utiles pour la distinguer d'autres maladies pulmonaires interstitielles chroniques.

reflecting a lack of standardized assessment or the presence of exposures that are not routinely acknowledged as causes of HP. The clinical utility of serum precipitins remains a source of debate and its diagnostic utility has not been validated in chronic HP. The diagnostic utility of lymphocyte transformation testing and professional home inspections remains unclear, and these are not routinely recommended (3). Specific provocation tests have been studied primarily in avian HP but a lack of standardized antigens and safety protocols limits its widespread use in clinical practice (6).

\section{Radiology}

Similar to other ILDs, the chest radiograph in HP may be normal or demonstrate reduced lung volumes with interstitial abnormalities. Whenever there is suspicion for ILD, a high-resolution computed tomography scan of the chest with expiratory views should be performed and interpreted by an experienced chest radiologist.

In acute or subacute HP, ground-glass opacities and centrilobular nodularity indicate active inflammation. Chronic HP is characterized by reticulation, traction bronchiectasis and, occasionally, honeycombing that can mimic usual interstitial pneumonia (UIP). Compared with IPF or NSIP, a mid-to-upper lung predominant or random pattern of ground-glass opacification or reticulation is suggestive of HP (7). HP is also often characterized by mosaic perfusion and air-trapping that is best identified on expiratory views. The juxtaposed combination of lobular areas of high, normal and low attenuation (the so-called 'head-cheese' sign) represents mixed interstitial and airways disease that can help distinguish HP from other fibrotic ILDs (8).

\section{Bronchoscopic and pathological examination}

Bronchoalveolar lavage fluid can be useful in supporting a diagnosis of HP as well as excluding other etiologies such as infection or hemorrhage. Increased bronchoalveolar lavage fluid cellularity with a lymphocytosis $>50 \%$ is strongly suggestive of HP in the appropriate clinical context, although its absence does not exclude the diagnosis, particularly in smokers and in patients with chronic HP (9). Transbronchial biopsies are rarely helpful to rule in chronic HP or

${ }^{1}$ Departments of Medicine; University of Calgary, Calgary, Alberta; ${ }^{2}$ University of California San Francisco, San Francisco; ${ }^{3}$ School of Public

Health, University of California, Berkeley, Berkeley, California, USA; ${ }^{4}$ Centre for Heart Lung Innovation $\mathcal{G}$ Department of Medicine,

University of British Columbia, Vancouver, British Columbia

Correspondence: Dr Kerri A Johannson, University of California, 505 Parnassus Avenue, San Francisco, California 94143, USA.

Telephone 415-502-2361, e-mail kerri.johannson@ucsf.edu 
TABLE 1

Key differences among hypersensitivity pneumonitis, idiopathic nonspecific interstitial pneumonia and idiopathic pulmonary fibrosis

\begin{tabular}{|c|c|c|c|}
\hline Characteristic & Hypersensitivity pneumonitis & Idiopathic nonspecific interstitial pneumonia & Idiopathic pulmonary fibrosis \\
\hline Typical age, years & Any (rare in children) & $40-60$ & $>50$ \\
\hline Sex ratio & Male $=$ female & Female $>$ male & Male $>$ female \\
\hline Chest HRCT & $\begin{array}{l}\text { Mid-to-upper lung predominant. Air- } \\
\text { trapping, mosaic perfusion, centrilobular } \\
\text { nodules, ground-glass opacities (may } \\
\text { have UIP pattern) }\end{array}$ & $\begin{array}{l}\text { Lower-lung predominant. Extensive ground-glass } \\
\text { opacities, mild reticulation, absence of } \\
\text { honeycombing, relative subpleural sparing }\end{array}$ & $\begin{array}{l}\text { Lower lung predominant. UIP pattern } \\
\text { (subpleural basilar predominant } \\
\text { reticulation, traction bronchiectasis and } \\
\text { honeycombing) }\end{array}$ \\
\hline Pathology & $\begin{array}{l}\text { Bronchiolocentric cellular interstitial fibrosis, } \\
\text { lymphocytic inflammation, poorly formed } \\
\text { granulomas (rarely UIP pattern) }\end{array}$ & Diffuse widening of the alveolar septa & $\begin{array}{l}\text { UIP pattern (fibroblast foci, microscopic } \\
\text { honeycombing, temporal heterogeneity) }\end{array}$ \\
\hline Management & Exposure remediation, immunosuppression & Immunosuppression & $\begin{array}{l}\text { Antifibrotic medications } \\
\text { (immunosuppression harmful) }\end{array}$ \\
\hline
\end{tabular}

HRCT High-resolution computed tomography; UIP Usual interstitial pneumonia

identify possible alternative diagnoses, although a normal transbronchial biopsy is also insufficient to exclude HP.

Surgical lung biopsies may show bronchiolocentric chronic interstitial pneumonia with poorly formed noncaseating granulomas, often with cholesterol clefts in the interstitium (10). An NSIP- or a UIPlike pattern with fibroblastic foci can be observed but will typically have other features suggesting HP. A classic pathological UIP pattern, even in the presence of a potential exposure, should prompt the clinician to consider IPF as the underlying diagnosis.

\section{Multidisciplinary evaluation}

Although several algorithms have been proposed $(2,4,6)$, there are no widely accepted diagnostic criteria for HP. The exposure history is an important component of multidisciplinary evaluation but lacks sufficient positive or negative predictive value to be useful in isolation. Patients with a clear exposure, compatible symptoms and supportive radiology do not require further evaluation. When the diagnosis remains uncertain after clinical and radiological assessment, bronchoscopic or pathological examination is required for a confident multidisciplinary diagnosis.

\section{MANAGEMENT}

The most important component of management is complete elimination of a suspected causative exposure (5). This can be financially or emotionally challenging, and any barriers to environmental remediation should be recognized early so that appropriate steps can be taken to ensure patient compliance. Appropriate patients with ILDassociated functional impairment should be referred for pulmonary rehabilitation. Lung transplantation may also be an option for patients with end-stage lung disease.

There are insufficient data to justify specific pharmacotherapy and, in this context, a therapeutic strategy based on the expected disease behaviour can be applied (11). Patients with mild disease and a clear exposure may not require systemic pharmacotherapy after complete removal of the inciting antigen. Patients with more advanced disease or with possible ongoing exposure may benefit from systemic corticosteroids and, possibly, steroid-sparing agents including mycophenolate mofetil, azathioprine and rituximab. The use of immunosuppressive and immunomodulatory medications is based primarily on case reports and small retrospective case series; their use should typically be considered only after assessment in a specialized ILD centre (12). The optimal duration of therapy is unknown and likely varies on a case-by-case basis; however, some patients may require several years of treatment.

\section{PROGNOSIS}

Chronic HP is typically slowly progressive, but can remain stable for prolonged periods or have episodes of acute exacerbation and rapid progression (13). Approximately $70 \%$ of patients with HP are alive 10 years after diagnosis, with early morbidity and mortality primarily occurring in patients with evidence of fibrosis (auscultatory crackles and radiological fibrosis) (2). A prediction model incorporating age, sex and lung physiology has been validated as a prognostic tool in HP (14). Identifying a specific exposure is also associated with improved outcomes, likely related to more complete elimination of the inciting antigen in these cases (5). Importantly, survival in HP is significantly longer than IPF, even with adjustment for the extent of fibrosis $(2,14)$.

\section{CONCLUSIONS AND FUTURE DIRECTIONS}

Chronic HP has a specific management and unique prognosis, emphasizing the importance of differentiating HP from other ILD subtypes. Accurate classification depends on a multidisciplinary review of clinical, radiological and pathological data when available. Management involves remediation of the identified exposure and immune suppression in patients at risk for progressive disease.

Further research is required to identify predisposing host factors, determine clinical and biological differences within subtypes of HP, and develop clinical prediction models for the diagnosis of HP. Widespread reporting of HP (eg, through a national registry) will enable geographical and spatial analysis to identify clustering of cases and previously unrecognized exposures. Randomized controlled trials of medical therapies are needed to guide management.

DISCLOSURES: The authors have no financial disclosures or conflicts of interest to declare.

\section{REFERENCES}

1. Lacasse Y, Girard M, Cormier Y. Recent advances in hypersensitivity pneumonitis. Chest 2012;142:208-17.

2. Mooney JJ, Elicker BM, Urbania TH, et al. Radiographic fibrosis score predicts survival in hypersensitivity pneumonitis. Chest 2013;144:586-92.

3. Selman M, Pardo A, King TE Jr. Hypersensitivity pneumonitis: Insights in diagnosis and pathobiology. Am J Respir Crit Care Med 2012;186:314-24.

4. Lacasse Y, Selman M, Costabel U, et al. Clinical diagnosis of hypersensitivity pneumonitis. Am J Respir Crit Care Med 2003;168:952-8.

5. Fernandez Perez ER, Swigris JJ, Forssen AV, et al. Identifying an inciting antigen is associated with improved survival in patients with chronic hypersensitivity pneumonitis. Chest 2013;144:1644-51.

6. Morell F, Villar A, Montero MA, et al. Chronic hypersensitivity pneumonitis in patients diagnosed with idiopathic pulmonary fibrosis: A prospective case-cohort study. Lancet Respir Med 2013;1:685-94.

7. Silva CI, Muller NL, Lynch DA, et al. Chronic hypersensitivity pneumonitis: Differentiation from idiopathic pulmonary fibrosis and nonspecific interstitial pneumonia by using thin-section CT. Radiology 2008;246:288-97. 
8. Chong BJ, Kanne JP, Chung JH. Headcheese sign. J Thorac Imaging 2014;29:W13.

9. Meyer KC, Raghu G, Baughman RP, et al. An official American Thoracic Society clinical practice guideline: The clinical utility of bronchoalveolar lavage cellular analysis in interstitial lung disease. Am J Respir Crit Care Med 2012;185:1004-14.

10. Jones KD, Urisman A. Histopathologic approach to the surgical lung biopsy in interstitial lung disease. Clin Chest Med 2012;33:27-40.

11. Travis WD, Costabel U, Hansell DM, et al. An official American Thoracic Society/European Respiratory Society statement: Update of the international multidisciplinary classification of the idiopathic interstitial pneumonias. Am J Respir Crit Care Med 2013;188:733-48

12. Lota HK, Keir GJ, Hansell DM, et al. Novel use of rituximab in hypersensitivity pneumonitis refractory to conventional treatment. Thorax 2013;68:780-1.

13. Churg A, Wright JL, Tazelaar HD. Acute exacerbations of fibrotic interstitial lung disease. Histopathology 2011;58:525-30.

14. Ryerson CJ, Vittinghoff E, Ley B, et al. Predicting survival across chronic interstitial lung disease: The ILD-GAP model. Chest 2014;145:723-8. 


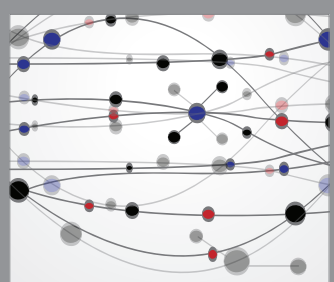

The Scientific World Journal
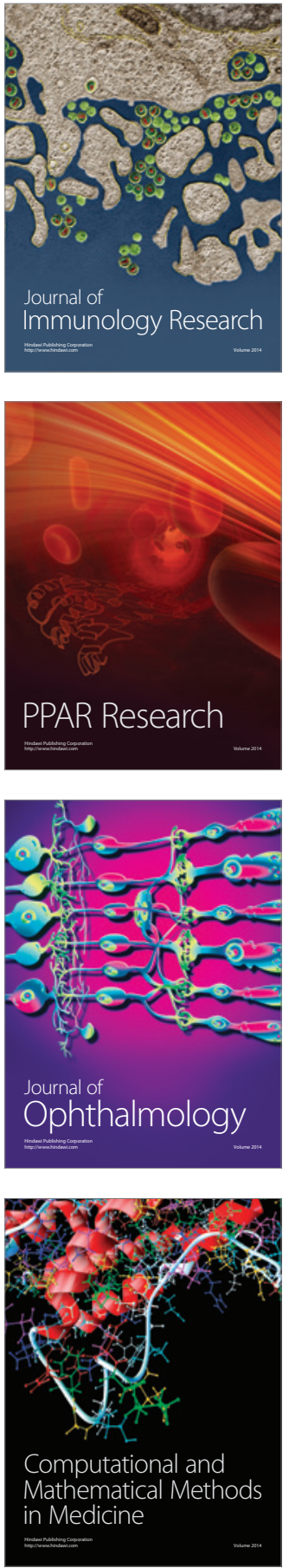

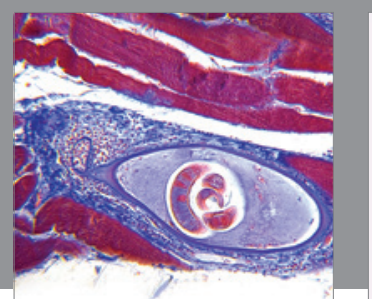

Gastroenterology Research and Practice

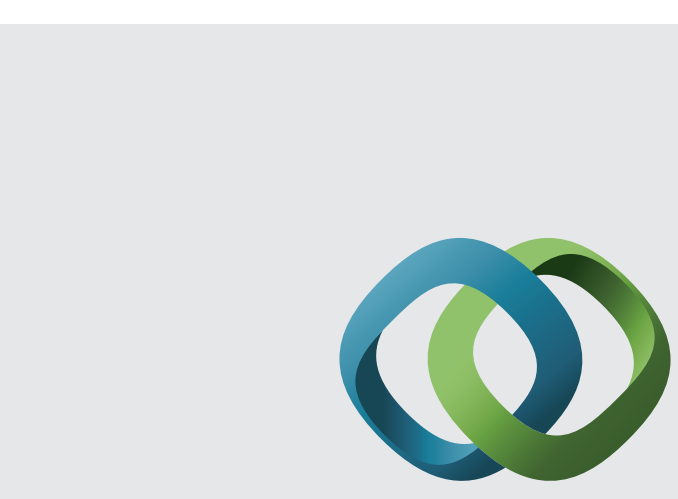

\section{Hindawi}

Submit your manuscripts at

http://www.hindawi.com
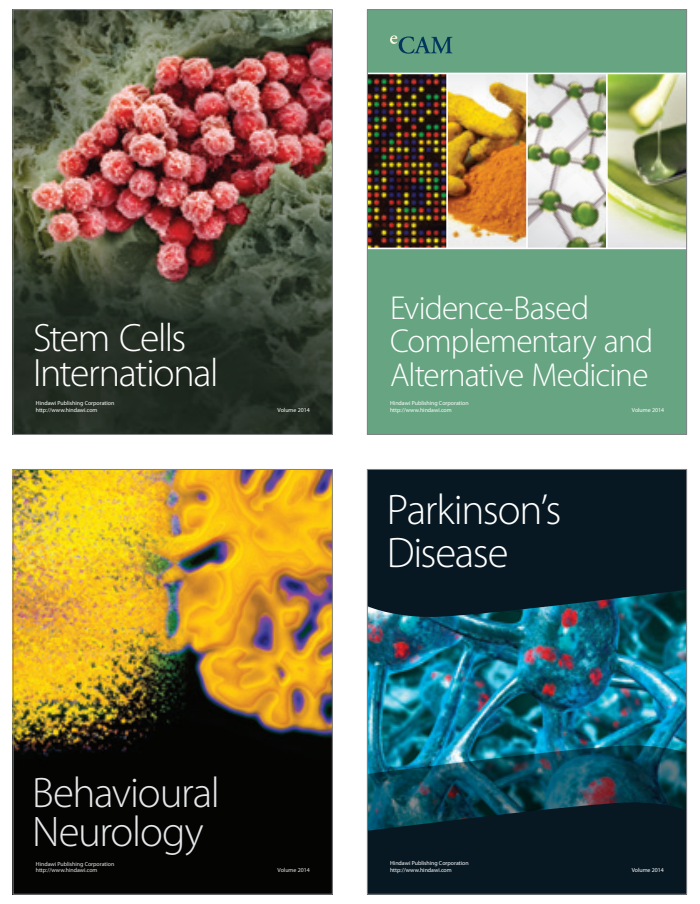
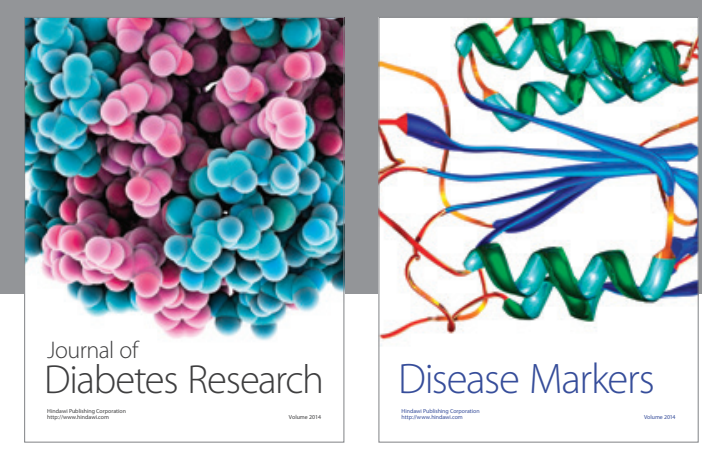

Disease Markers
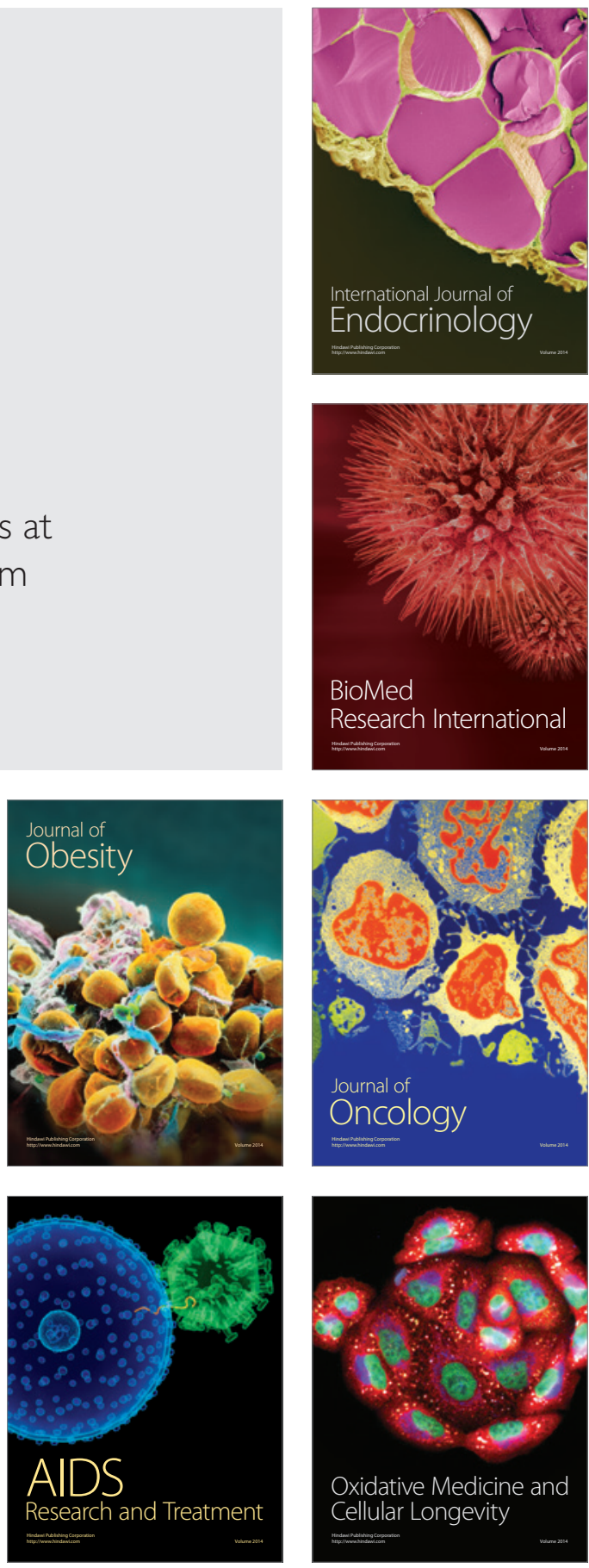